\title{
SiGe BiCMOS for Analog, High-Speed Digital and Millimetre-Wave Applications Beyond $50 \mathrm{GHz}$
}

\author{
S.P. Voinigescu, T. Chalvatzis, K.H.K. Yau, A. Hazneci, A. Garg, S. Shahramian, T. Yao, \\ M. Gordon, T.O. Dickson, E. Laskin, S.T. Nicolson, A.C. Carusone, L. Tchoketch-Kebir, \\ O. Yuryevich, G. Ng, B. Lai, and P. Liu
}

ECE Dept., University of Toronto, 10 King's College Rd., Toronto, ON, M5S 3G4, Canada

\begin{abstract}
This paper explores the application of SiGe BiCMOS technology to mm-wave transceivers with analog and digital signal processing. A review of 1080Gb/s SERDES performance across $3 \mathrm{SiGe}$ BiCMOS and CMOS technology nodes reveals remarkable similarities with digital CMOS IC scaling and points to the benefits of a SiGe BiCMOS roadmap. Examples of 40-Gb/s equalizers, track-and-hold amplifiers and ADCs with $\mathbf{m m}$-wave sampling clocks are provided, along with GHz-range opamp filters and $65-\mathbf{G H z}$ wireless transceivers. Automotive radar and imaging applications in the 80-100 $\mathrm{GHz}$ range are also briefly discussed.
\end{abstract}

\section{INTRODUCTION}

Historically, due to larger-scale economies, wireless and (briefly) fibre-optic applications have driven SiGe BiCMOS process development [1]-[3]. While SiGe HBT performance has steadily improved over the last few years [4]-[8], the frequency of most applications has remained in the $2-10 \mathrm{GHz}$ range. This has allowed CMOS technology to catch up in some of the larger volume markets, such as WLAN and $10-\mathrm{Gb} / \mathrm{s}$ datacom, where SiGe BiCMOS technology had carved a niche. The shift away from continual speed enhancement and towards higher digital signal processing content will place greater demand in the future for ultra-fast ADC techniques in applications such as $40-\mathrm{Gb} / \mathrm{s}$ fibre-optic, backplane and chip-tochip communication, instrumentation, and digital cinema. In these applications, SiGe HBTs must be paired with nanoscale MOSFETs to deliver the required performance at acceptable power dissipation levels. Ultra high $f_{\mathrm{T}}$ and $f_{\mathrm{MAX}}$ values, simultaneously exceeding $250 \mathrm{GHz}$, as well as low phase noise are, however, required for the potentially lucrative $77 / 94 \mathrm{GHz}$ automotive, mm-wave imaging, and 100-Gb/s Ethernet applications. This paper addresses transistor performance scaling and building blocks for $40-80 \mathrm{~Gb} / \mathrm{s}$ transceivers with analog and digital signal processing and for mm-wave radio and radar.

\section{SiGE HBT AND SERDES PERFORMANCE SCALING}

Benefiting from the clear guidelines set forth by the International Roadmap for Semiconductors (ITRS),
CMOS technology scaling has continued unabated to nanometre dimensions. Cut-off and maximum oscillation frequencies in general purpose (GP) 65-nm CMOS technologies with physical gate lengths below $45 \mathrm{~nm}$ now exceed $200 \mathrm{GHz}$ at $1 \mathrm{~V}$ supply [9]. Power dissipation, noise figure, and phase noise performance in CMOS RF and fibre-optic ICs improve with scaling. At the same time, as the data compiled in Fig. 1 illustrate, SiGe BiCMOS technology evolution has been rather irregular, with missed nodes and at least 3 foundries jockeying up for the leading position at one time or another. Interestingly, a clear scaling law exists, as indicated by the dashed trend line, which allows $\mathrm{SiGe}$ BiCMOS to retain a 2-generation advantage over CMOS in terms of $f_{\mathrm{T}}$ and $f_{\mathrm{MAX}}$.

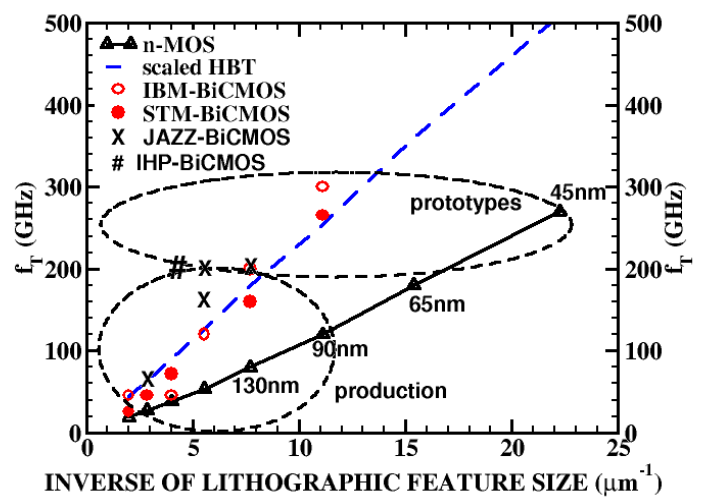

Fig. $1 f_{\mathrm{T}}$ scaling in CMOS and SiGe BiCMOS technology nodes

Fig. 2 shows the measured $f_{\mathrm{T}}$ of production and of prototype SiGe HBTs as a function of collector current density, along with the $f_{\mathrm{T}}$ of a scaled device simulated using Silvaco's T-CAD platform. The scaled transistor has 100-nm emitter width, a 5-nm thick base doped $8 \times 10^{19} \mathrm{~cm}^{-3}$, a $25-\mathrm{nm}$ thick collector, and features a trapezoidal Ge profile varying from $20 \%$ to $40 \%$. Its $N F_{M I N}$ is $1.1 \mathrm{~dB}$ at $65 \mathrm{GHz}$ while $f_{\mathrm{T}}$ and $f_{\text {MAX }}$ simultaneously exceed $500 \mathrm{GHz}$ at a current density of $30 \mathrm{~mA} / \mu \mathrm{m}^{2}$ with a peak of $560 \mathrm{GHz}$ at $60 \mathrm{~mA} / \mu \mathrm{m}^{2}$. The simulator was calibrated on experimental $160-\mathrm{GHz}$ SiGe HBT characteristics from production $\mathrm{SiGe}$ BiCMOS processes [4], [6]. For comparison, an InP HBT with 12.5-nm base width and $710 / 340 \mathrm{GHz} f_{\mathrm{T}} / f_{\mathrm{MAX}}$ at $20 \mathrm{~mA} / \mu \mathrm{m}^{2}$ was 
recently fabricated [10]. These results indicate that a SiGe HBT structure, much like the ones prototyping today, is capable of maintaining the $2 \times$ advantage over 45-nm MOSFETs. However, the significant increase in current density with scaling, and slightly higher $N F_{M I N}$ than MOSFETs at comparable $f_{\mathrm{T}}$, remain weak points of SiGe HBTs. Indeed, experimental device [11] and $60-\mathrm{GHz}$ circuit data [12] indicate comparable or lower $N F_{M I N}$ for $90 / 65-$ nm MOSFETs than for the best SiGe HBTs.

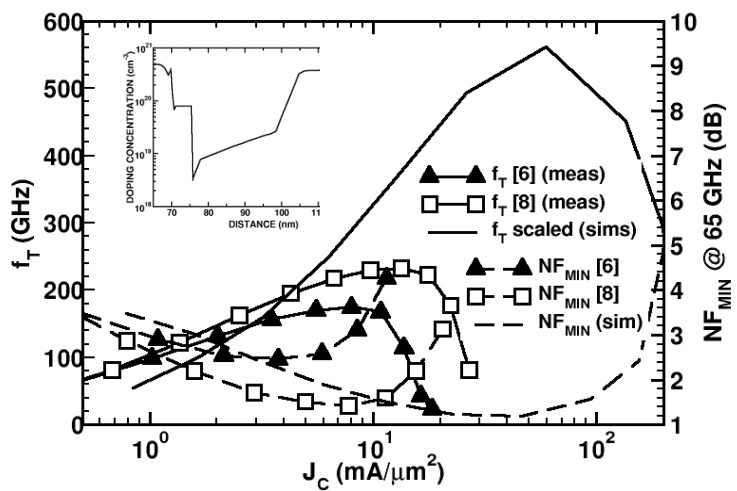

Fig. $2 f_{\mathrm{T}}$ and $N F_{M I N}$ at $65 \mathrm{GHz}$ vs $J_{c}$ in production [6], prototype [8], and in simulated scaled SiGe HBTs. The simulated structure is shown in the inset.

A fully integrated 5-GHz wireless transceiver and a 10-Gb/s SONET receiver were first reported at MRS in 1998 [13]. These circuits were implemented in an early version of a $0.5 \mu \mathrm{m}$ SiGe BiCMOS process with $45 / 60-\mathrm{GHz} f_{\mathrm{T}} / f_{\mathrm{MAX}}$ [1]. The SONET receiver employed $E^{2} \mathrm{CL}$ logic and operated from a $-5.2 \mathrm{~V}$ supply. The first single-chip $10-\mathrm{Gb} / \mathrm{s}$ SONET SERDES [14] and a 10-Gb/s Ethernet (10GE) SERDES (Fig. 3) fabricated in $0.35 \mu \mathrm{m}$ [2] and $0.25 \mu \mathrm{m}$ [3] SiGe BiCMOS processes were introduced in December 2000 and the summer of 2001, respectively. Both these ICs rely on simpler ECL families to reduce the supply voltage of the $10-\mathrm{Gb} / \mathrm{s}$ circuitry to $3.3 \mathrm{~V}$.

The $0.35 \mu \mathrm{m}$ SiGe HBT has an $f_{\mathrm{T}}$ of $45 \mathrm{GHz}$ and allows for a $10-\mathrm{GHz}$ latch to be realized with $2-\mathrm{mA}$ tail current and no inductive peaking. The total power dissipation of the SONET SERDES is $2.2 \mathrm{~W}$ and includes a VCSEL driver with output swing and preemphasis control, laser bias and monitoring circuitry. The output swing and VCSEL modulating current are adjustable over a $3: 1$ range up to $2 \times 750 \mathrm{mV}_{\mathrm{pp}}$ and $15 \mathrm{~mA}$, respectively [14]. The 10GE SERDES dissipates $3.2 \mathrm{~W}$ from $3.3-\mathrm{V}, 2.5-\mathrm{V}$ and $1.8-\mathrm{V}$ supplies. It incorporates over 200,000 digital CMOS gates embedded between the analog $10.3-\mathrm{Gb} / \mathrm{s}$ and 3.125-Gb/s interfaces (Fig.3). Taking advantage of both SiGe HBT and CMOS scaling across technology nodes, and reducing the number of vertically-stacked transistors, two new generations of this chip have since been ported, first to $180-\mathrm{nm}$ SiGe BiCMOS [4] dissipating about $2 \mathrm{~W}$ from $3.3-\mathrm{V}$ and $1.8-\mathrm{V}$ supplies, and next to $130-n m$ CMOS, dissipating approximately $1 \mathrm{~W}$ from a $1.2-\mathrm{V}$ supply.

The most interesting aspect of this SERDES family is that it demonstrates the benefits of scaling for mixed-signal SiGe BiCMOS products. Scaling leads to an almost linear improvement in jitter, rise/fall times, die area, supply voltage and power dissipation [15]. It also confirms the prediction made in [14] that only at the $130-\mathrm{nm}$ node would CMOS be able to compete with SiGe BiCMOS technology for $10-\mathrm{Gb} / \mathrm{s}$ SERDES applications. Indeed, several $10-\mathrm{Gb} / \mathrm{s}$ SERDES chips in 130-nm CMOS, including a VCSEL driver [16], have been reported during the last 2 years while 180-nm CMOS solutions could not compete [17].

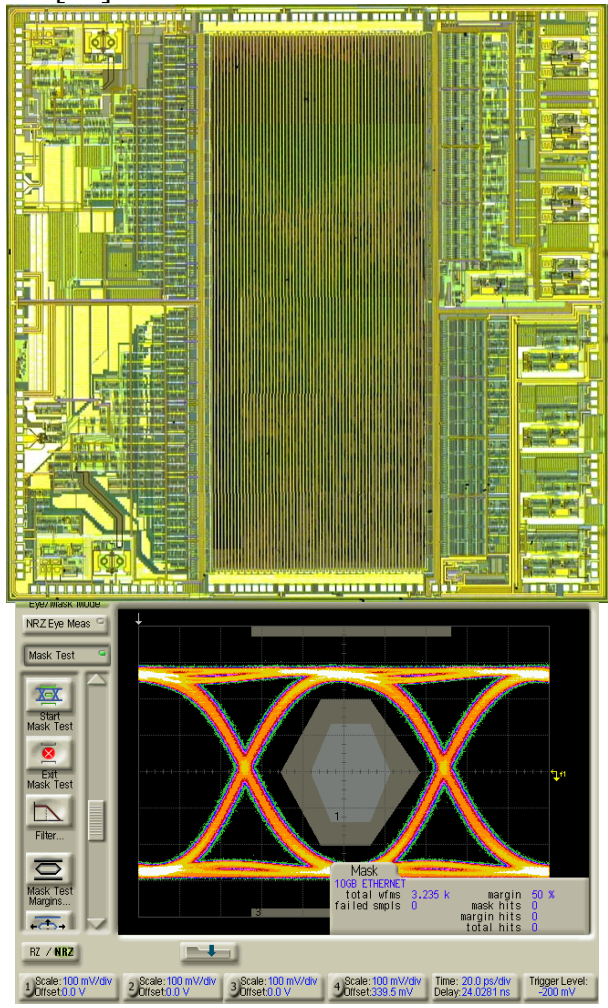

Fig. 3 Single-chip 10GE transceiver in $0.25 \mu \mathrm{m} \mathrm{SiGe}$ BiCMOS: a) die photograph and b) $10.3-\mathrm{Gb} / \mathrm{s}$ output eye diagram (Quake Technologies 2001)

Although a 40-GHz flip-flop [18] and a $60-\mathrm{Gb} / \mathrm{s} \mathrm{2:1}$ MUX [19] have recently been realized in 90-nm CMOS, a $45-\mathrm{GHz}$ flip-flop, as needed for a full-rate 40-Gb/s SONET SERDES with FEC, has only been demonstrated in InP [20] and $\mathrm{SiGe}$ BiCMOS technologies [21]-[23]. Scaled 40/80-Gb/s SERDES can now be fabricated in 130-nm SiGe BiCMOS technology [6], operating from $2.5-\mathrm{V}$ supply with similar power dissipation and performance margin (Fig.4) as the first-generation 10GE chip (Fig. 3). This (4-8) $\times$ speed increase [23] at comparable power dissipation and cost has been made possible by the $4 \times$ improvement in transistor $f_{\mathrm{T}} / f_{\mathrm{MAX}}$ and by relying on a MOS-SiGe HBT cascode topology and inductive 
peaking [23]. The combination of MOSFETs and HBTs on the high-speed path capitalizes on the low $V_{T}$ of nanoscale MOSFETs, the cancellation of Miller capacitance, and on the low gate resistance to improve switching speed over HBT-only logic while reducing the power supply voltage to $2.5 \mathrm{~V}$ and $1.8 \mathrm{~V}[11,24]$.

Fig. 5 illustrates the scaling of MOS-HBT cascode inverter delay between production $180-\mathrm{nm}$ and 130nm SiGe BiCMOS nodes with comparable SiGe HBT performance. The benefits of future scaling to $90-\mathrm{nm}$ BiCMOS are also clearly apparent where the delay reduction from $5.1 \mathrm{ps}$ to $3.3 \mathrm{ps}$ is solely due to replacing 130-nm with 90-nm MOSFETs. The inset shows how emitter-followers (EF) can be employed for level shifting and further increase speed. Note that the optimal delay is achieved when the tail current corresponds to $0.3 \mathrm{~mA} / \mu \mathrm{m}$ of gate width for the MOSFETs that make up the differential pair [27].

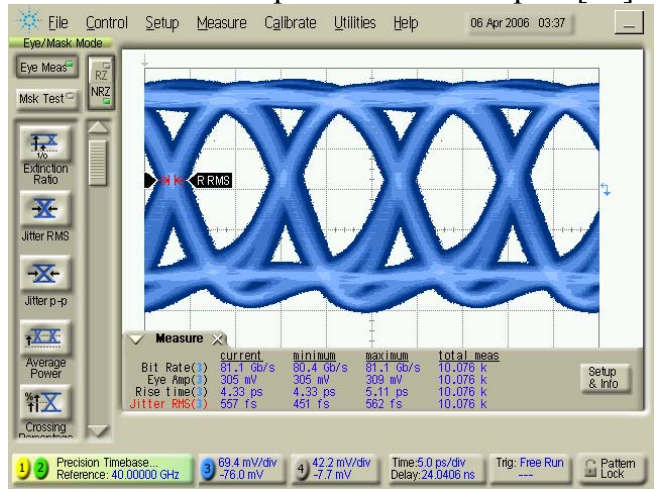

Fig. $42 \times 300 \mathrm{mV}_{\mathrm{pp}}, 80-\mathrm{Gb} / \mathrm{s}$ output eye diagram (running for 1 hour) of a $2.5-\mathrm{V}, 1.4 \mathrm{~W}$ transmitter [23] implemented in a $130-\mathrm{nm} \mathrm{SiGe} \mathrm{BiCMOS}$ process [6].

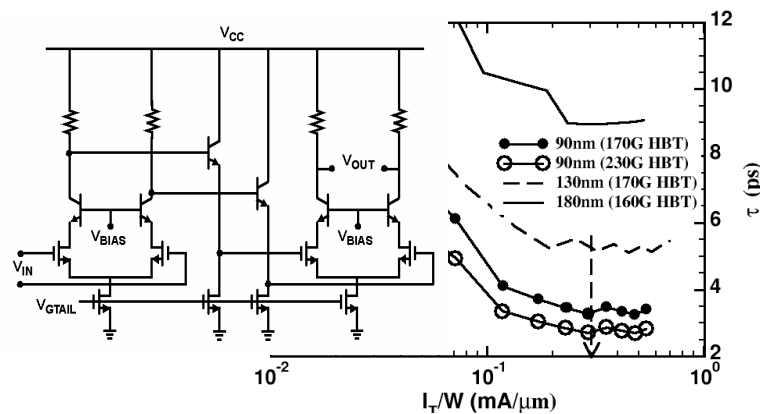

Fig. 5 Scaling of BiCMOS cascode inverter delay across nodes obtained from measured DC and high-frequency characteristics. All inverters have a gain of 1.5 and a fanout of 1. The impact of the EF stage is not included.

\section{ANALOG CIRCUITS}

Operational amplifiers (opamps) can be used in GHz-range filters and delta-sigma modulators if their unity gain bandwidth exceeds $10 \mathrm{GHz}$ [25], [26]. The schematic of a fully differential opamp employing a MOS-HBT cascode with cascode p-MOSFET load [25] is shown in Fig. 6. The circuit has an EF output for level-shifting purposes and to reduce the output impedance. It was implemented in 130-nm and 180$\mathrm{nm}$ generations of SiGe BiCMOS technologies with similar HBT $f_{\mathrm{T}} / f_{\mathrm{MAX}}$ [25],[26]. The MOS-HBT cascode has speed, linearity and noise advantages over the HBT-HBT cascode. This is the result of the lower input time constant $\mathrm{R}_{\mathrm{G}} \times\left(\mathrm{C}_{\mathrm{gs}}+\mathrm{C}_{\mathrm{gd}}\right)$, better phase margin and concomitant low-noise and high-linearity bias in MOSFETs, as opposed to HBTs. To maximize the unity gain bandwidth, all MOSFETs and HBTs are biased at the peak $f_{\text {MAX }}$ current density which, for n-MOSFETs and p-MOSFETs, is approximately $0.2 \mathrm{~mA} / \mu \mathrm{m}$ and $0.08 \mathrm{~mA} / \mu \mathrm{m}$, respectively, across technology nodes and foundries [27]. A record unity gain bandwidth of $37 \mathrm{GHz}$ was measured in the 130-nm half-circuit with $10-\mathrm{mA}$ current [25]. Opamp biquad filters (Fig. 7) were fabricated and their performance was compared with that of 2-stage $\mathrm{g}_{\mathrm{m}}$-LC bandpass filters based on the same MOS-HBT cascode topology (Fig. 8). The measured transfer characteristics, $N F$ and linearity of both filters are shown in Figs. 9-11. With comparable linearity and power dissipation, the opamp filter occupies 10 times smaller area than the $\mathrm{g}_{\mathrm{m}}-\mathrm{LC}$ filter.

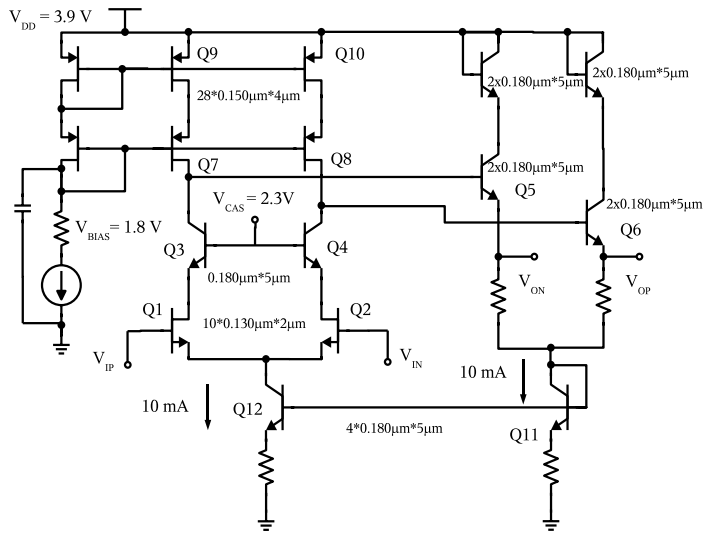

Fig. 6 130-nm SiGe BiCMOS opamp schematics.

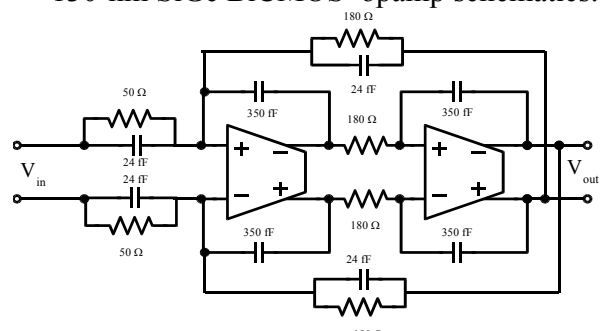

Fig. 7 130-nm SiGe BiCMOS biquad filter diagram.

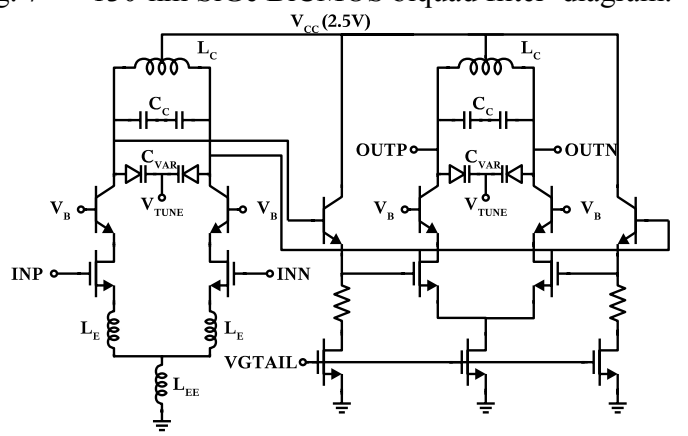

Fig. 8 Block diagram of 130-nm SiGe BiCMOS $\mathrm{g}_{\mathrm{m}}$-LC filter based on a BiCMOS cascode. 


\section{40-GB/s EQUALIZERS}

Production SiGe BiCMOS technology is wellpoised to address $45-\mathrm{Gb} / \mathrm{s}$ transceivers with equalization. A typical block diagram of an equalizer with analog DSP is shown in Fig. 12. While the FFE block could be realized in $90-\mathrm{nm}$ CMOS, the DFE requires a full-rate $45-\mathrm{GHz}$ flip-flop which is unlikely to be implemented in CMOS with adequate margin before the $65-\mathrm{nm}$ node.

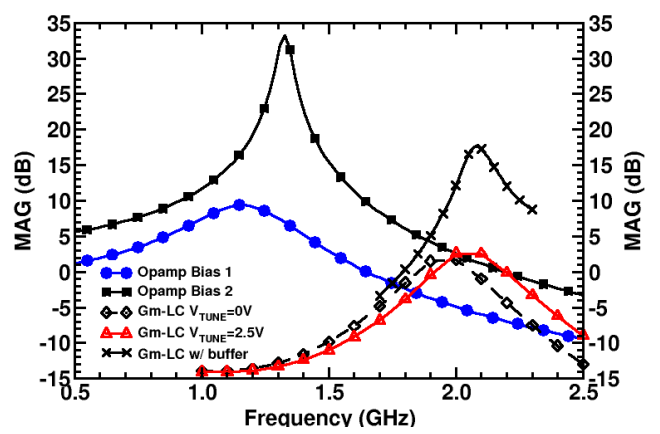

Fig. 9 Measured biquad and gm-LC filter characteristics for different bias conditions. Measurements of the $\mathrm{g}_{\mathrm{m}}-\mathrm{LC}$ filters with and without an output buffer are provided.

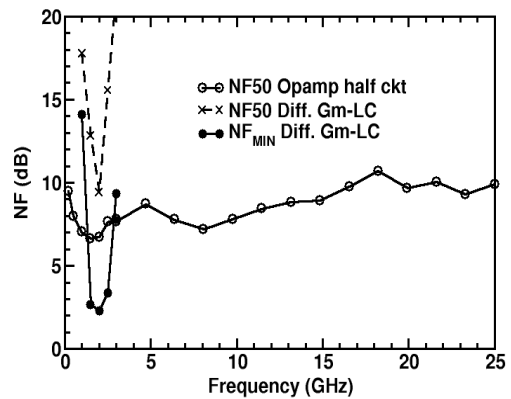

Fig. 10 Measured 50- $\Omega N F$ of opamp half-circuit and of differential gm-LC filter.

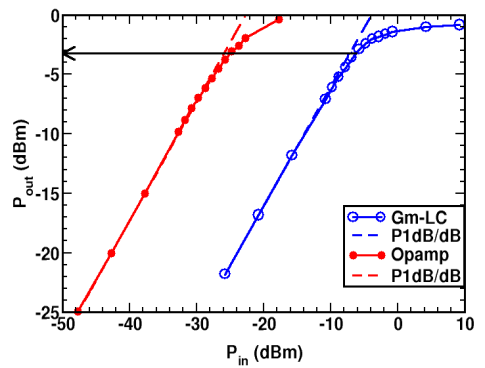

Fig. 11 Comparison of the measured linearity of twostage opamp and $\mathrm{g}_{\mathrm{m}}-\mathrm{LC}$ filters operating at $1.2 \mathrm{GHz}$ and 1.9 $\mathrm{GHz}$, respectively.

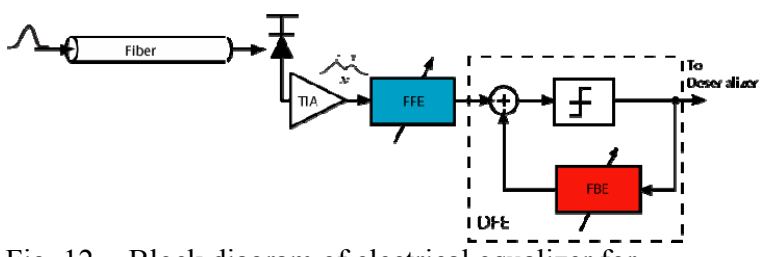

Fig. 12 Block diagram of electrical equalizer for polarization mode dispersion in optical fibers.

\section{A. Feed Forward Equalizer}

Fig. 13 describes the block diagram of a 7-tap 40-Gb/s FFE [28] implemented in a $180-\mathrm{nm}$ SiGe BiCMOS process [4]. A differential, distributed topology is employed which is realized with M6-overM2 microstrip lines and HBT-cascode taps with gain and sign control. The measured and modelled characteristics of the 9-ft SMA cable used in systemlevel simulations and experiments are illustrated in Figs. 14-16. The circuit is capable of compensating for more than $20 \mathrm{~dB}$ loss at $20 \mathrm{GHz}$. Error-free operation was verified from $5 \mathrm{~Gb} / \mathrm{s}$ to $40 \mathrm{~Gb} / \mathrm{s}$, and equalized eyes were obtained up to $49 \mathrm{~Gb} / \mathrm{s}$ [28].

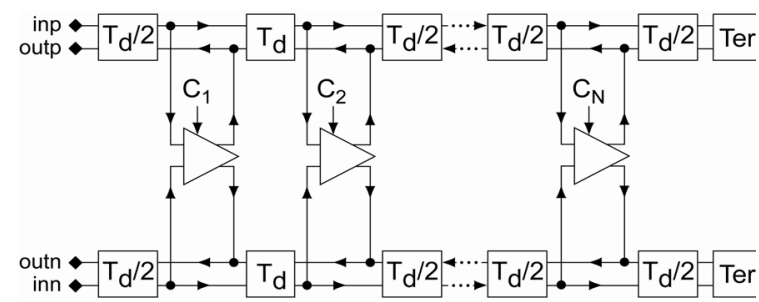

Fig. 13 Schematics of 7-tap FFE implemented in 180-nm SiGe BiCMOS.

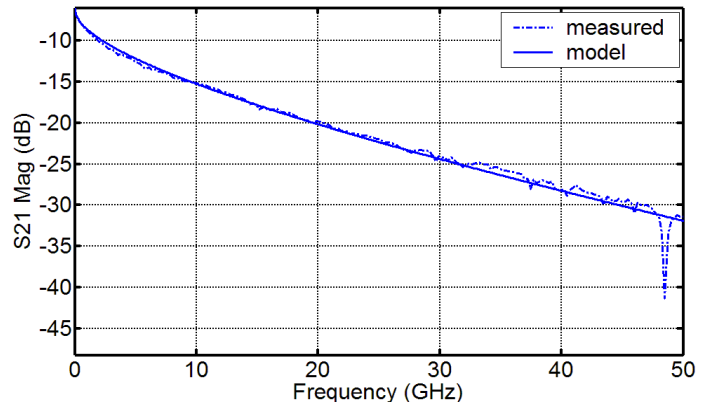

Fig. 14 Modeled vs. measured transmission loss of 9-ft SMA cable.
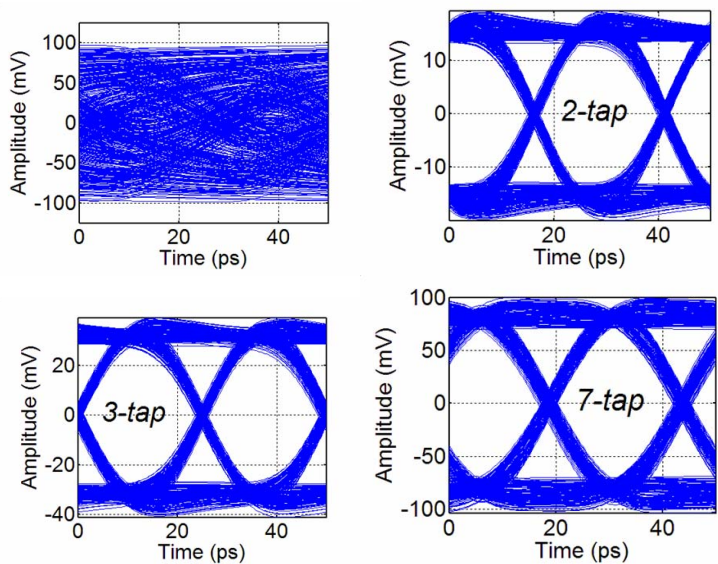

Fig. 15 Modelled 40-Gb/s equalized eye diagrams as a function of the number of equalizer taps. a) input signal after passing through the SMA cable and output of b) 2-tap, c) 3-tap, d) 7-tap equalizers.

\section{B. $40 \mathrm{~Gb} / \mathrm{s} \mathrm{DFE}$}

The DFE whose block diagram is shown in Fig. 17, was implemented in the same technology [4] with 3.3-V ECL and verified to correctly equalize a PRBS 
passing through a 9-ft SMA cable at data rates from $5 \mathrm{~Gb} / \mathrm{s}$ to $39.5 \mathrm{~Gb} / \mathrm{s}$, Fig.18 [29].

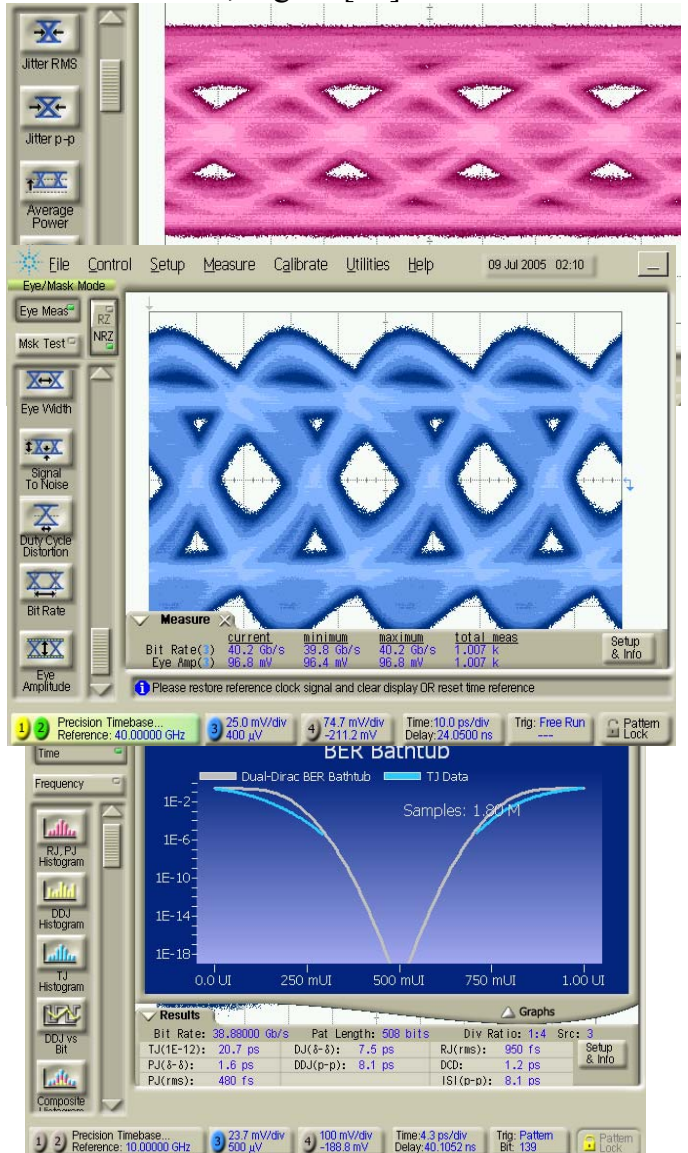

Fig. 16 FFE input/output $40-\mathrm{Gb} / \mathrm{s}$ eye diagrams and bathtub.

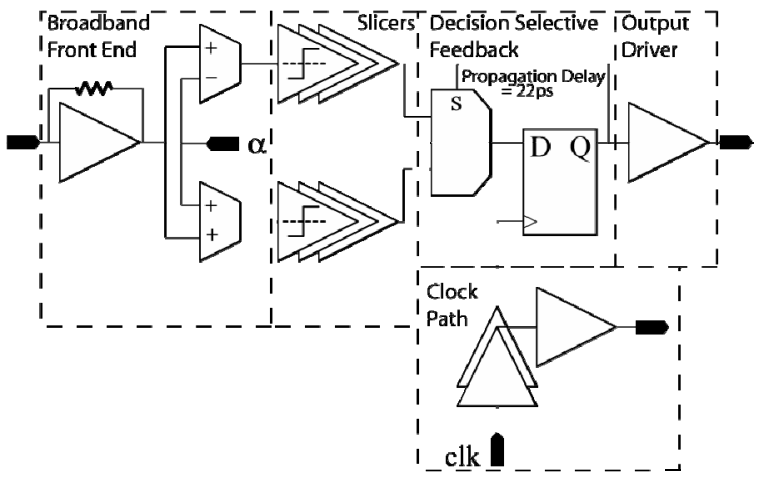

Fig. 17 1-tap look-ahead DFE architecture.

\section{C. $40 \mathrm{~Gb} / \mathrm{s}$ Digital Equalizer Blocks}

When the technology has adequate speed, a digital DSP solution (Fig. 19) is preferred over an analog one due to its increased flexibility. In this architecture, the most critical block is the T\&H amplifier (Fig. 20), which must simultaneously satisfy broad band, low noise and high linearity requirements. A T\&H amplifier with over $40-\mathrm{GHz}$ bandwidth (Fig. 21) and 4.5-bit linearity was reported in [30].

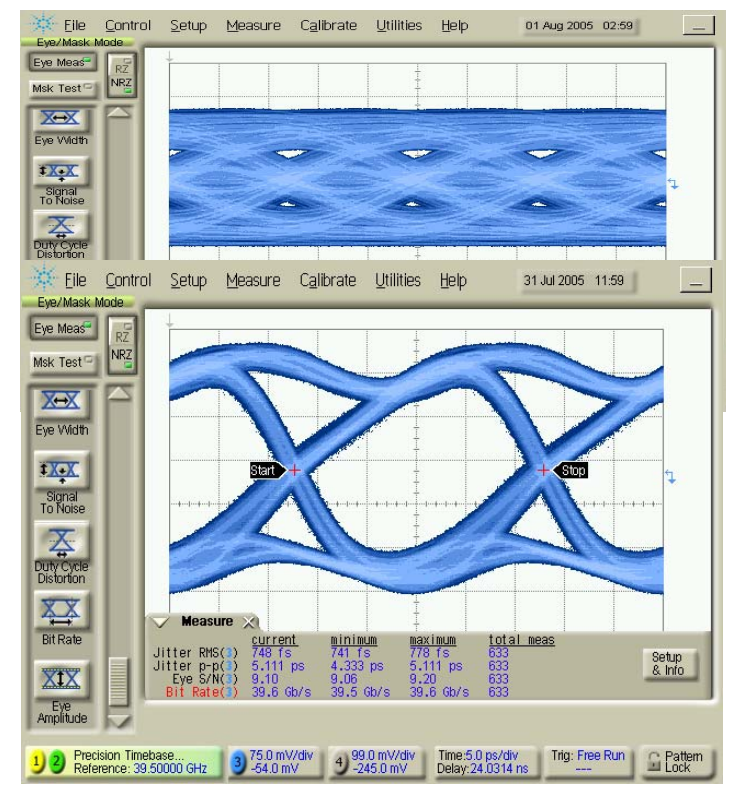

Fig. 18 39.5-Gb/s DFE input and output eye diagrams after passing through a 9-ft long SMA cable.

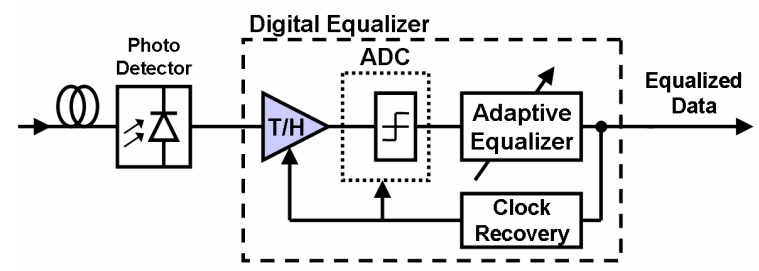

Fig. 19 Block diagram of a DSP based fiber optic equalizer.

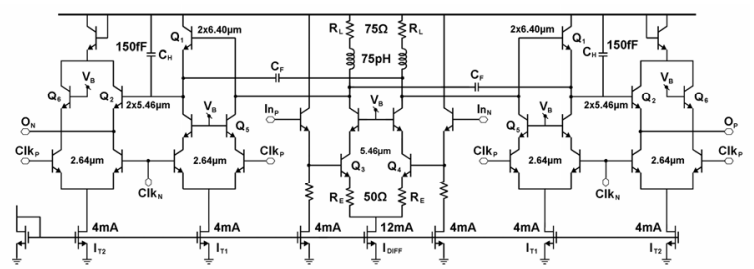

Fig. 20 Circuit diagram of the track \& hold block in 180nm SiGe BiCMOS [4]. Signals $\mathrm{In}_{\mathrm{N}}$ and $\mathrm{In}_{\mathrm{P}}$ are provided by a low-noise, broadband transimpedance amplifier [30].

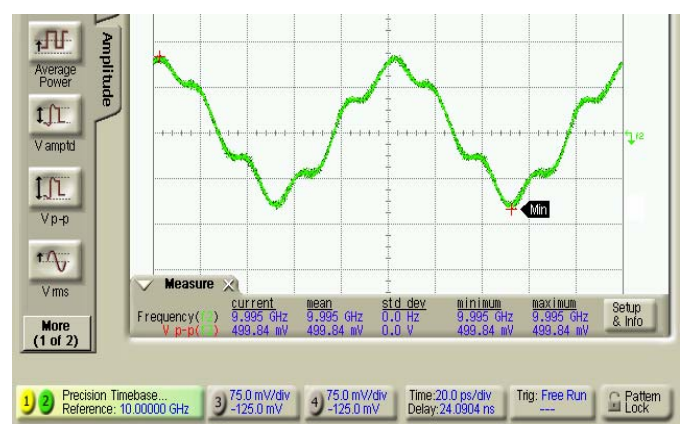

Fig. 21 Differential output of the T\&H amplifier for an $10-\mathrm{GHz}$ sinusoid sampled at $40 \mathrm{GHz}$ (70 $\mathrm{mV} / \mathrm{div})$ [30].

\section{ADCs}

The high intrinsic speed of both SiGe HBTs [1]-[8] and CMOS [11], [12] transistors provides an excellent 
incentive for the introduction of digital signal processing techniques relying on high oversampling ratios and mm-wave clock frequencies. Such techniques typically require only simple circuit topologies, similar to those used in a fiberoptic SERDES, that can be designed to be robust to process variations, transistor leakage and non-linearity. The 2-GHz bandpass $\Delta \Sigma$ design described in [31] employs the $\mathrm{g}_{\mathrm{m}}$-LC transconductor from Fig. 8 and the BiCMOS cascode MSM flip-flop [22] as the 40GSamples/sec quantizer. The IIP3 test in Fig. 23 shows an SFDR of $61 \mathrm{~dB}$ with an ENOB of 8.5 over a $120 \mathrm{MHz}$ band centred at $2 \mathrm{GHz}$. The $40-\mathrm{Gb} / \mathrm{s}$ output stream, reproduced in Fig. 24, can be decimated with the same 2.5-V CML BiCMOS logic family [22]-[24].

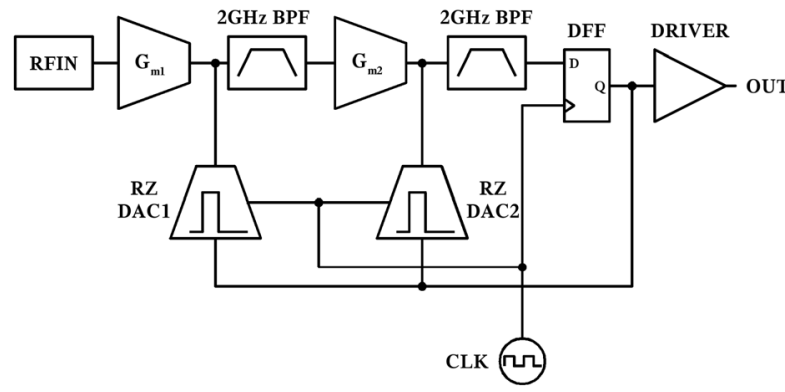

Fig. 22 System level $\Delta \Sigma$ ADC diagram [31]

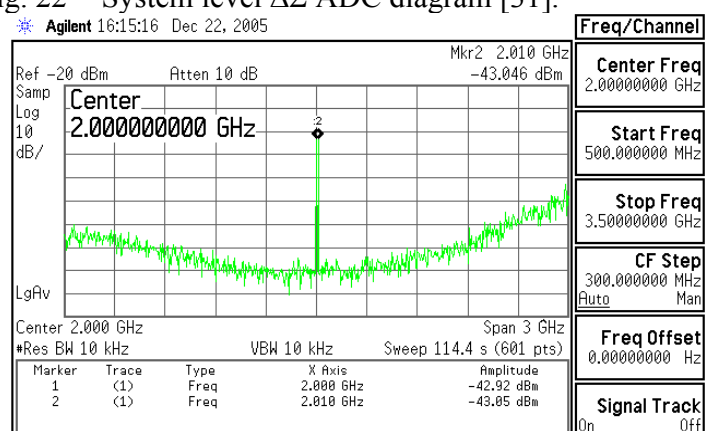

Fig. 23 IIP3 test for 40-GS/s 2-GHz bandpass ADC.

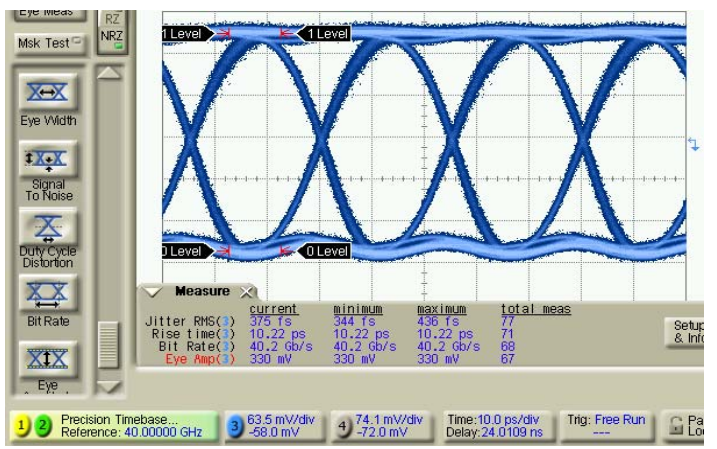

Fig. $24 \Delta \Sigma$ bandpass ADC output eye diagram in open loop at $40 \mathrm{~Gb} / \mathrm{s}$ with a $2-\mathrm{GHz}$ sinusoidal input.

\section{Millimetre WaVe Transceivers}

Figs. 25 and 26 reproduce the schematics and die photos of a $60-\mathrm{GHz}$ WLAN transceiver chip set and of a $65-\mathrm{GHz}$ Doppler sensor with on-die patch antenna [32], [33] implemented in a production 180nm SiGe BiCMOS process with $f_{\mathrm{T}} / f_{\mathrm{MAX}}$ of $160 \mathrm{GHz}$
[4]. The transmitter has an image-reject architecture with stagger-tuned, two-stage, lumped poly-phase filters at $5 \mathrm{GHz}$ and at $65 \mathrm{GHz}$. All three ICs include for the first time a low-phase-noise $60-\mathrm{GHz} \mathrm{VCO}$ with $10 \%$ tuning range [34] and rely on inductors and transformers to minimize die area. The VCO, downconvert mixer, LNA, and power amplifier employ SiGe HBT cascodes with inductive degeneration to improve isolation and simplify interstage matching. The entire design process was conducted using hand analysis and Spectre, as in the 2-10 $\mathrm{GHz}$ range [35], while inductors and transformers were designed using ASITIC.
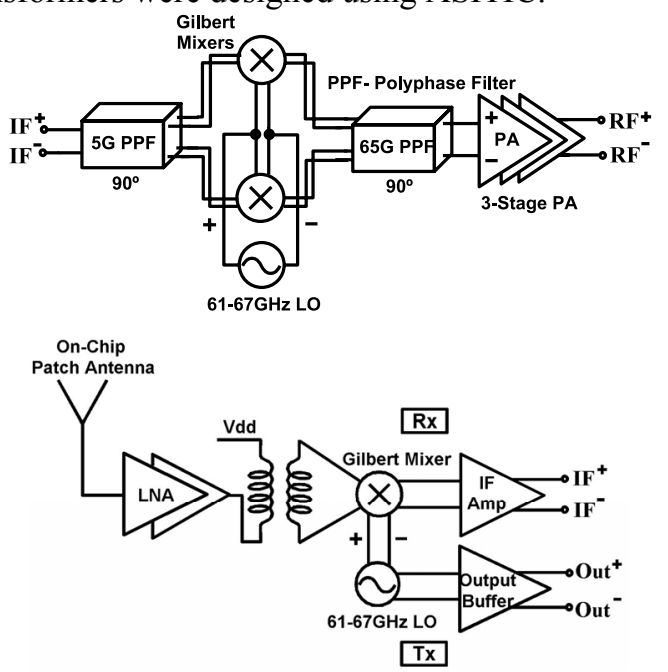

Fig. 25 Block diagrams of a) 60-GHz radio transmitter, b) Doppler radar implemented in 180-nm SiGe BiCMOS.

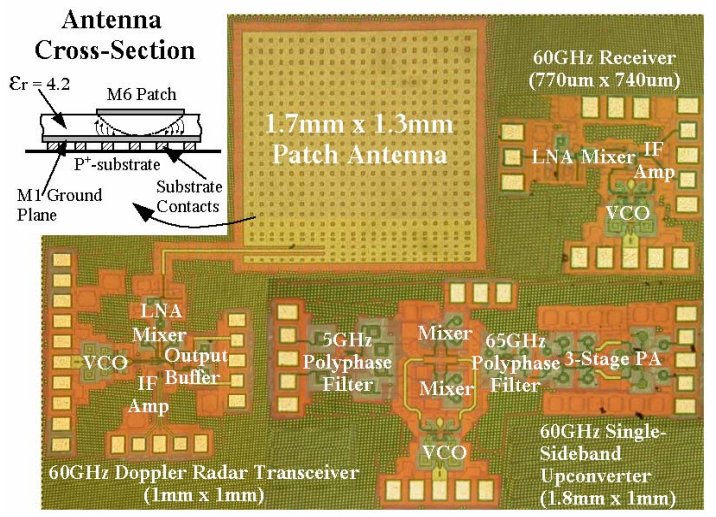

Fig. 26 Die photos of Doppler radar with patch antenna (left) WLAN transmitter (bottom) and receiver (top right).

Fig. 27 shows the measured conversion gain of the WLAN receiver measured on wafer, and of the Doppler sensor with and without the on-die antenna. The Doppler sensor with antenna was tested either by placing a horn antenna, or a GGB probe at different elevations above the on-die antenna. The doublesideband (DSB) noise figure, typically $12 \mathrm{~dB}$, and the corresponding single-ended down-conversion gain of the WLAN receiver, $15 \mathrm{~dB}$ at $2.5 \mathrm{~V}$, and $20 \mathrm{~dB}$ at 3.3 $\mathrm{V}$ supply, are compiled in Fig. 28. More than $45 \mathrm{~dB}$ of image rejection has been measured in the WLAN transmitter, Fig. 29. 


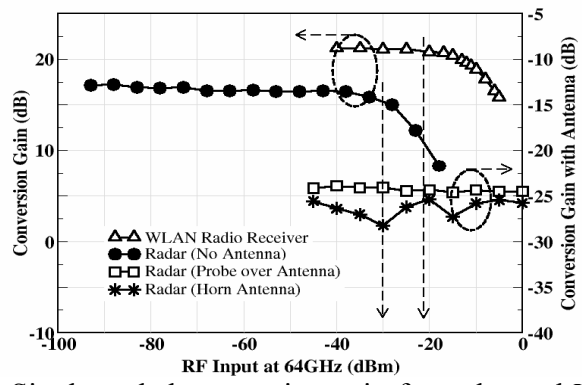

Fig. 27 Single-ended conversion gain for radar and WLAN receiver with radio IF at $1 \mathrm{GHz}$ and radar IF at $730 \mathrm{MHz}$.

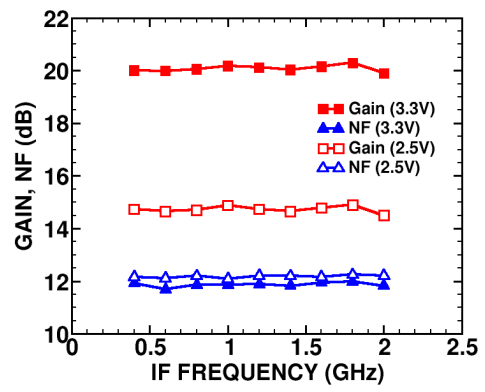

Fig. 28 Measured WLAN receiver gain and noise figure.

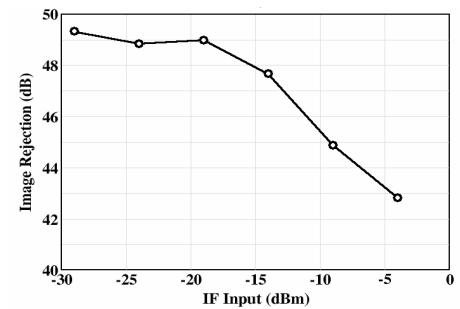

Fig. 29 SSB WLAN radio transmitter image rejection vs. IF input power with $61-\mathrm{GHz} \mathrm{LO}$ and 5-GHz IF.

Even though prototype SiGe HBTs and production 65-nm MOSFETs [12] now have adequate performance for automotive radar and imaging at 94 $\mathrm{GHz}$ [36],[37], on-chip isolation remains a major concern and will influence the choice of system architecture. For example, beyond $100 \mathrm{GHz}$, antennas are small enough for phase arrays to be integrated in silicon but crosstalk will limit their applicability. Alternatively, rectangular waveguide arrays are reasonably small, have excellent isolation and, in conjunction with finlines, provide more efficient radiation. For mm-wave medical imaging, where arrays of tens of transceivers are required, low (phase) noise [36], isolation and power dissipation are the most important design goals. Fig. 30 reproduces the measured $N F_{M I N}$ at $5 \mathrm{GHz}$ and at $65 \mathrm{GHz}$ in HBTs with $f_{M A X}$ of $200 \mathrm{GHz}$ and $300 \mathrm{GHz}$, respectively. $N F_{\text {MIN }}$ was obtained from Y-parameter measurements as in [35],[39]. The impact of transistor vertical profile scaling on $N F_{M I N}$ is negligible at $5 \mathrm{GHz}$ but becomes significant at $65 \mathrm{GHz}$. Furthermore, since the transit time through the collector space charge region is significantly larger than the base transit time, correlation between the collector and base shot noise currents increases, pushing the optimum noise current density at mm-waves close to the peak $f_{\mathrm{T}} / f_{\mathrm{MAX}}$ current density and changing the value of the optimum noise impedance. The latter has important implications for LNA and VCO design, and allowed us to achieve a record phase noise of $-101.3 \mathrm{dBc} / \mathrm{Hz}$ at $1 \mathrm{MHz}$ from the 103.6 GHz carrier, as shown in Fig. 31 [36].

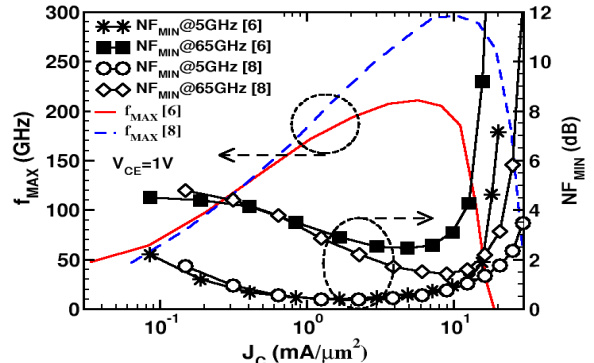

Fig. 30 Measurements of $\mathrm{NF}_{\mathrm{MIN}}$ scaling in SiGe HBTs.

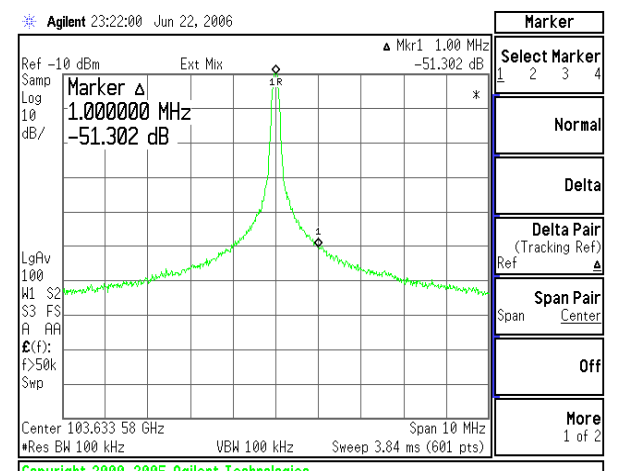

Fig. 31 Measured spectrum, phase noise of 103-GHz VCO.

\section{VII.CONCLUSIONS}

Low-frequency analog figures of merit such as $g_{m} / I$, $g_{m} / g_{o}$, lower $1 / f$ noise and the larger $f_{\mathrm{T}} \times \mathrm{BV}_{\mathrm{CEO}}$ product remain the main strengths of SiGe HBTs well into the $\mathrm{mm}$-wave regime. However, historical data, moderate volume markets and relentless pressure from more aggressively scaled CMOS point to the importance of pairing next generation $250 / 500 \mathrm{GHz}$ SiGe HBTs with 90-nm CMOS as a viable and economical way to capture all applications demanding fast HBTs and at least some of the $90 / 65-\mathrm{nm}$ CMOS market. In that scenario the discontinued supply-voltage scaling of 65-nm CMOS will help release most of the pressure off SiGe. Stand-alone SiGe HBT technology, stripped of its CMOS advantage, will have a difficult time competing with InP or CMOS.

\section{ACKNOWLEDGEMENTS}

We thank NSERC, Micronet, CITO, Gennum, NORTEL and STM for funding, and STM and Jazz Semiconductor for fabrication. Equipment grants from CFI and OIT, and CAD tools from CMC are also acknowledged. We are grateful to Dan Trepanier of Quake for the 10GE SERDES data, and to Pascal Chevalier and Alain Chantre of STM for $230-\mathrm{GHz}$ HBT discussions. S. Voinigescu would like to thank 
Rudy Beerkens, Bernard Sautreuil, Paul Kempf and Marco Racanelli for their support over many years, and his former colleagues at NORTEL and Quake Technologies in Ottawa, Canada.

\section{REFERENCES}

[1] D.L. Harame et al., "Si/SiGe Epitaxial-Base Transistors -Part II: Process Integration and Analog Applications," IEEE Trans. ED, Vol.42, pp. 459-482, 1995.

[2] A. Monroy, et al., "A high performance $0.35 \mu \mathrm{m}$ SiGe BiCMOS technology for wireless applications," Proc. IEEE BCTM, pp.121-124, Sept. 1999.

[3] H. Baudry et al., "High performance $0.25 \mu \mathrm{m}$ SiGe and SiGe:C HBTs using non-selective epitaxy," Proc. IEEE BCTM, pp.52-55, Sept. 2001

[4] M. Racanelli, et al., "Ultra High Speed SiGe NPN for advanced BiCMOS technology," IEEE IEDM Techn. Digest, pp. 336-339, Dec. 2001.; M. Racanell and P. Kempf, "SiGe BiCMOS Technology for RF Circuit Applications," IEEE Trans. ED, Vol.52, pp. 1259$1270,2005$.

[5] B. Jaganathan, et al. "Self-aligned SiGe NPN transistors with $285 \mathrm{GHz} f_{\mathrm{MAX}}$ and $207 \mathrm{GHz} f_{\mathrm{T}}$ in a manufacturable technology," IEEE Electron Device Lett., Vol.23, No.5, pp.258-260, March. 2002.

[6] M. Laurens et al, "A $150 \mathrm{GHz} f_{\mathrm{T}} / f_{\mathrm{MAX}} 0.13 \mu \mathrm{m} \mathrm{SiGe:C}$ BiCMOS technology," Proc. IEEE BCTM, pp.199-202, Sept. 2003.

[7] T.F. Meister et al., "SiGe Bipolar Technology with 3.9ps gate delay,"IEEE BCTM, pp.103-106, Sept. 2003.

[8] P. Chevalier et al, "300-GHz $f_{\text {MAX }}$ self-aligned $\mathrm{SiGeC}$ HBT optimized towards CMOS compatibility," Proc. IEEE BCTM, pp.120-123, Oct. 2005.

[9] B. Jagannathan, et al, "RF CMOS for Microwave and MM-wave Applications," IEEE SiRF, Techn. Digest. pp.259-264, Jan. 2006.

[10] W. Hafez et al., "12.5 nm base pseudomorphic heterojunction bipolar transistors achieving $f_{\mathrm{T}}=710$ $\mathrm{GHz}$ and $f_{\mathrm{MAX}}=340 \mathrm{GHz}$," Appl.Phys.Lett., Vol.87, pp.252109-1/3, Dec. 2005

[11] P. Chevalier et al., "Advanced SiGe BiCMOS and CMOS Platforms for Optical and mm-wave ICs," IEEE CSICS, San Antonio, Nov. 2006.

D. Grindberg, "Silicon Heterostructure Handbook," pp.4.5-439-457 Taylor \& Francis, 2006.

[12] T. Yao, et al., "60-GHz PA and LNA in 90-nm RFCMOS," IEEE RFIC Symposium Digest, pp. 147-150, June 2006.

D. Alldred et al. "A $1.2 \mathrm{~V}, 60 \mathrm{GHz}$ radio receiver with on-chip transformers and inductors in 90nm CMOS," IEEE CSICS, San Antonio, Nov. 2006.

[13] R. Hadaway, et al., "Application of SiGe HBTs to Datacom and Wireless," MRS, April, 1998.

[14] S.P. Voinigescu et al., "Circuits and Technologies for Highly Integrated Optical Networking ICs at $10 \mathrm{~Gb} / \mathrm{s}$ to 40Gb/s," Proc. IEEE CICC, pp.331-338, May 2001.

[15] D. Trepanier, IEEE CSICS Panel Nov. 2005.

[16] S. Rabii, et al., "An Integrated VCSEL Driver for 10 $\mathrm{Gb} / \mathrm{s}$ Ethernet in $0.13 \mu \mathrm{m}$ CMOS," IEEE ISSCC Digest, pp.246-247, Feb. 2006.

[17] J. Cao et al, "OC-192 Receiver in Standard $0.18 \mu \mathrm{m}$ CMOS," ISSCC Digest, pp.250-251, Feb. 2002.

[18] T. Chalvatzis, et al., "A $40 \mathrm{~Gb} / \mathrm{s}$ Decision Circuit in 90-nm CMOS," ESSCIRC, Montreux, Sept. 2006.

[19] D. Kehrer et al., "A $60 \mathrm{~Gb} / \mathrm{s} 2: 1$ Selector in $90 \mathrm{~nm}$ CMOS," IEEE CSICS Digest, pp.105-108, Oct. 2004.
[20] A. Hendarman, et al., "STS-768 Multiplexer With FullRate Output Data Retimer in InP HBT," IEEE J.SolidState Circuits, Vol.38, pp.1497-1503, Sept. 2003.

[21] M. Meghelli, "A 108Gb/s 4:1 Multiplexer in $0.13 \mu \mathrm{m}$ SiGe-Bipolar Technology," ISSCC Digest, pp.236-237, Feb. 2004

[22] T.O. Dickson, et al., "A 2.5-V, 45-Gb/s Decision Circuit Using SiGe BiCMOS Logic," IEEE J. SolidState Circuits, Vol.40, No.4, pp.994-1003, 2005.

[23] T.O. Dickson and S. P. Voinigescu., "Low-power circuits for a 10.7 -to- $86 \mathrm{~Gb} / \mathrm{s} 80-\mathrm{Gb} / \mathrm{s}$ serial transmitter in 130-nm SiGe BiCMOS," IEEE CSICS, Nov. 2006.

[24] E. Laskin and S.P. Voinigescu, "A $60 \mathrm{~mW}$ per Lane, 4 $\times 23-\mathrm{Gb} / \mathrm{s} 2^{7}-1$ PRBS Generator," IEEE CSICS, Techn. Digest, pp.192-195, Nov. 2005.

[25] S.P. Voinigescu, et al., "Design Methodology and Applications of SiGe BiCMOS Cascode Opamps with up to 37-GHz Unity Gain Bandwidth," IEEE CSICS, Techn. Digest, pp.283-286, Nov. 2005.

[26] G. Ng, et al., "1 GHz Opamp-Based Bandpass Filter," IEEE SiRF, Techn. Digest. pp.369-372, Jan. 2006.

[27] T.O. Dickson et al., "The Invariance of Characteristic Current Densities in Nanoscale MOSFETs and its Impact on Algorithmic Design Methodologies and Design Porting of $\mathrm{Si}(\mathrm{Ge})$ (Bi)CMOS High-Speed Building Blocks," IEEE J. S-St. Circuits, Aug. 2006.

[28] A. Hazneci and S. P. Voinigescu, "49-Gb/s, 7-Tap Transversal Filter in $0.18 \mu \mathrm{m}$ SiGe BiCMOS for Backplane Equalization," IEEE CSICS, pp.101-104, Oct.2004.

[29] A. Garg, et al., “A 1-Tap 40-Gbs Lookahead Decision Feedback Equalizer in $0.18 \mu \mathrm{m}$ SiGe BiCMOS Technology," IEEE CSICS, pp.37-41, Nov.2005.

[30] S. Shahramian, et al., "A 40-GSamples/Sec Track \& Hold Amplifier in $0.18 \mu \mathrm{m}$ SiGe BiCMOS Technology," IEEE CSICS, pp.101-104, Nov. 2005.

[31] T. Chalvatzis and S. P. Voinigescu, "A Low-Noise 40-GS/s Continuous-Time Bandpass $\Delta \Sigma$ ADC Centered at 2GHz," IEEE RFIC Symposium Digest, pp. 323-326, June 2006.

[32] M. Gordon, et al.,"65-GHz Receiver in SiGe BiCMOS Using Monolithic Inductors and Transformers" IEEE SiRF Techn. Digest. pp.265-268, Jan. 2006.

[33] T. Yao, et al., "65GHz Doppler Radar Transceiver with On-Chip Antenna in $0.18 \mu \mathrm{m}$ SiGe BiCMOS," IEEE IMS Digest, pp.1493-1496, June 2006.

[34] C. Lee, et al., "SiGe BiCMOS 65-GHz BPSK Transmitter and 30 to $122 \mathrm{GHz}$ LC-Varactor VCOs with up to $21 \%$ Tuning Range," IEEE CSICS, Technical Digest, pp.179-182, Oct. 2004.

[35] S.P. Voinigescu et al, "A scalable high-frequency noise model for bipolar transistors with application to optimal transistor sizing for low-noise amplifier design," IEEE J. Solid-State Circuits, vol. 32, pp. 1430-1438, Sept. 1997.

[36] S.T. Nicolson, et al, "Design and Scaling of SiGe BiCMOS VCOs Operating near $100 \mathrm{GHz}$," IEEE BCTM 2006.

[37] E. Laskin et al. "Low-Power, Low-Phase Noise SiGe HBT Static Frequency Divider Topologies up to $100 \mathrm{GHz}, "$ IEEE BCTM 2006.

[38] T.O. Dickson, S.P. Voinigescu, "SiGe BiCMOS Topologies for Low-Voltage Mm-Wave VoltageControlled Oscillators and Frequency Dividers," IEEE SiRF, Techn. Digest. pp.273-276, Jan. 2006.

[39] K.H.K. Yau and S.P. Voinigescu, "Modeling and Extraction of SiGe HBT Noise Parameters from Measured Y-Parameters and Accounting for Noise Correlation,"IEEE SiRF Digest. pp.226-229, Jan. 2006. 